\title{
Choroidal Lymphoma Discovered on Ultrasound in a Patient with Suspected Corneal Tumor
}

\author{
Christos Theophanous $^{\mathrm{a}}$ Melike Pekmezci $^{\mathrm{a}}$ b Bertil E. Damato $^{\mathrm{a}}$
}

Andrew A. Kao ${ }^{a}$ Michele M. Bloomer ${ }^{\mathrm{a}}$ Jay M. Stewart ${ }^{\mathrm{a}}$ Armin R. Afshar ${ }^{\mathrm{a}}$

a Department of Ophthalmology, University of California, San Francisco, CA, USA; ${ }^{b}$ Department of Pathology,

University of California, San Francisco, CA, USA

\section{Established Facts}

- Choroidal lymphoma is a rare ocular tumor.

- Diagnosis may be delayed due to mildness of symptoms.

- Ultrasound is the most useful imaging modality; histopathologic confirmation is required for definitive diagnosis.

\section{Novel Insights}

- Choroidal lymphoma in the context of corneal scarring has not been previously reported.

- Corneal scarring may prevent visualization of the posterior segment and increases the importance of $\mathrm{B}$-scan identification of choroidal thickening in discovering underlying lymphoma.

\section{Keywords}

Uveal lymphoma · Choroidal lymphoma · Ocular oncology

\begin{abstract}
Background/Aims: To report the case of a 77-year-old male with a blind, painful eye, referred for suspected corneal mass, with finding of choroidal B-cell lymphoma on pathology of enucleated globe. Methods: This is a retrospective case report of a single patient. Results: A 77-year-old male with a longstanding history of poor vision in the left eye was
\end{abstract}

referred for a scarred, vascularized corneal mass. The patient had reported occasional mild ocular discomfort in the left eye and loss of light perception over the last year. Visual acuity was 20/20 in the right eye and no light perception in the left eye. Intraocular pressure was $32 \mathrm{~mm} \mathrm{Hg}$ in the left eye. Fundoscopic visualization was not possible due to corneal opacity. B-scan ultrasound showed an infiltrative, low-reflective choroidal lesion and inferior retinal detachment. Pathology from the enucleated globe revealed diffuse sheets of CD20+ small B cells replacing the choroid, characteristic of a low-grade small B-cell extranodal marginal zone lymphoma.

\section{KARGER}

(c) 2018 S. Karger AG, Basel

E-Mail karger@karger.com

www.karger.com/oop
Armin R. Afshar, MD, MBA

Department of Ophthalmology, University of California, San Francisco

10 Koret Way, K-304, Box 0730

San Francisco, CA 94131 (USA)

E-Mail Armin.Afshar@ucsf.edu 
Conclusion: This is an unusual presentation of choroidal lymphoma in an eye with severe corneal opacification and scarring, and underscores the diagnostic value of ultrasonography in examination of eyes without view to the posterior segment.

(c) 2018 S. Karger AG, Basel

\section{Introduction}

Choroidal lymphoma is a rare ocular disease characterized by the proliferation of lymphoid cells, typically B cells, into the choroid tissue [1]. Cases can be classified into either primary lymphomas, which are the least common and are typically low-grade B-cell non-Hodgkin's lymphomas, or secondary manifestations of systemic lymphomas, which are more often higher-grade malignancies [2]. Although few large studies have been conducted on the disease due to its rarity, several reports have identified clinical patterns in choroidal lymphoma patients. Choroidal lymphomas are typically unilateral and present with mild, insidious onset of symptoms (if any), such as blurred vision, with few patients reporting ocular pain or redness [3]. Compared to primary lymphomas, secondary lymphomas were more likely to be bilateral and are associated with poorer visual acuity [3]. The mildness of symptoms with choroidal lymphoma can delay diagnosis.

On clinical exam, the presence of multifocal, creamyyellow patches at the level of the choroid on fundoscopic exam, obscuration of the choroidal blood vessels, and anterior transscleral epibulbar extensions visible as pink masses under the conjunctiva and Tenon's fascia are characteristic [3]. Ultrasound is the most useful imaging modality; hollow thickening of the choroid and posterior epibulbar extensions can be seen, with low-reflectivity in the lesions [3]. Histopathologic confirmation is required for definitive diagnosis, which can be achieved using transvitreal or direct transscleral needle or vitrectomy cutter biopsy [2-4]. Herein, we describe a case of a choroidal lymphoma identified during workup for a suspected corneal mass, confirmed through histopathologic analysis of the enucleated globe.

\section{Case Presentation}

A 77-year-old man with a 10-year history of poor vision in the left eye was referred to ocular oncology clinic for a suspected corneal mass. The patient reported that a corneal scar had developed 10 years earlier and had been attributed to a herpes simplex virus (HSV) infection. In the 3 months prior, the corneal scar progressed

Choroidal Lymphoma on Ultrasound in a

Patient with Suspected Corneal Tumor

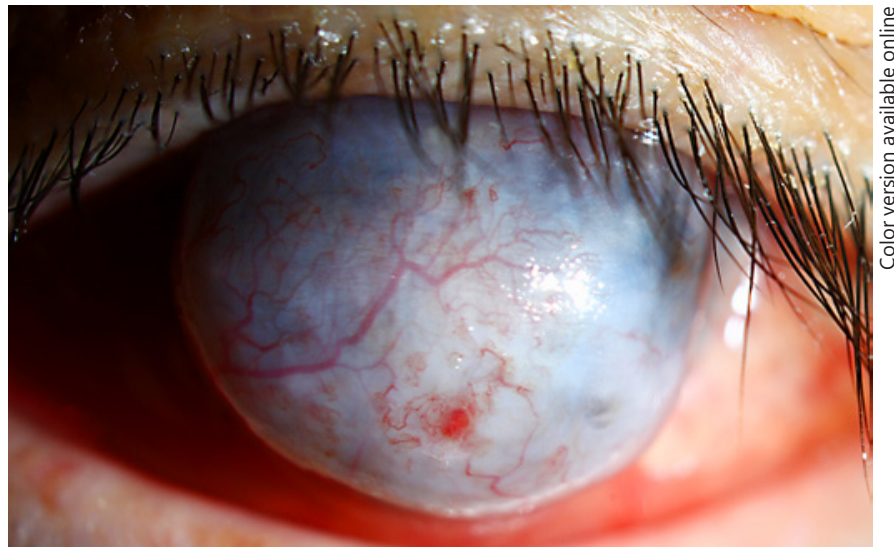

Fig. 1. Slit-lamp image of left eye: descemetocele with corneal opacification, scarring, and vascularization.

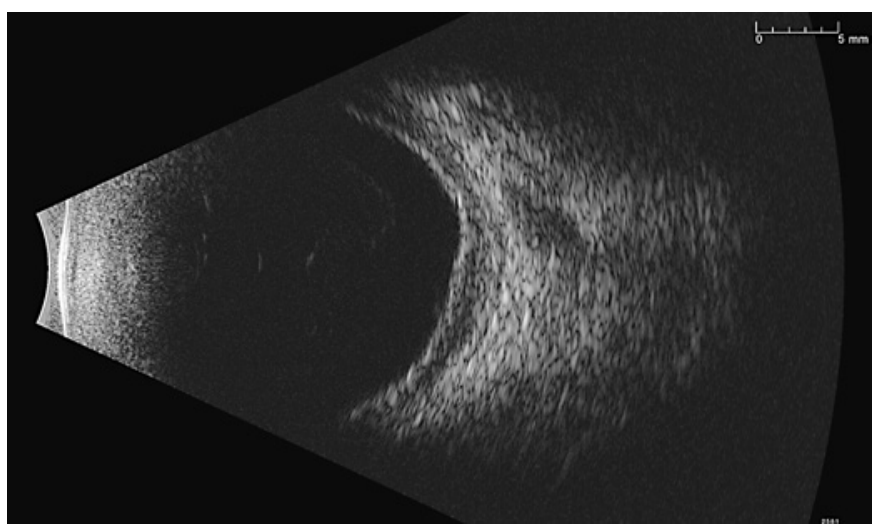

Fig. 2. B-scan ultrasonography of the left eye showing infiltrative choroidal lesion.

and bulged into what appeared as a mass. Over the previous year, the patient experienced occasional mild discomfort and had gradually lost the ability to perceive light and shadows (the patient's baseline). The patient had no other significant ocular history. His medical history was significant for prostate cancer treated surgically, and coronary artery disease treated with bypass surgery and pacemaker implantation.

On examination, visual acuity was $20 / 20$ in the right eye and no light perception in the left eye. Intraocular pressure by pneumatonometry was $20 \mathrm{~mm} \mathrm{Hg}$ in the right eye and $32 \mathrm{~mm} \mathrm{Hg}$ in the left eye. Extraocular movements were full bilaterally. Slit-lamp examination of the left eye revealed moderate conjunctival injection of the left eye with a large central ectatic area and descemetocele protruding $5 \mathrm{~mm}$ from the corneal surface, which was opaque and vascularized, obscuring any view of the anterior or posterior segments (Fig. 1). Examination of the right eye was unremarkable. B-scan ultrasonography of the left eye was performed and revealed an infiltrative, lowreflective choroidal lesion with inferior retinal detachment (Fig. 2).

The patient elected for an enucleation with a $22-\mathrm{mm}$ silicone sphere implant (Bausch \& Lomb, Inc., Irvine, CA, USA). Histo- 

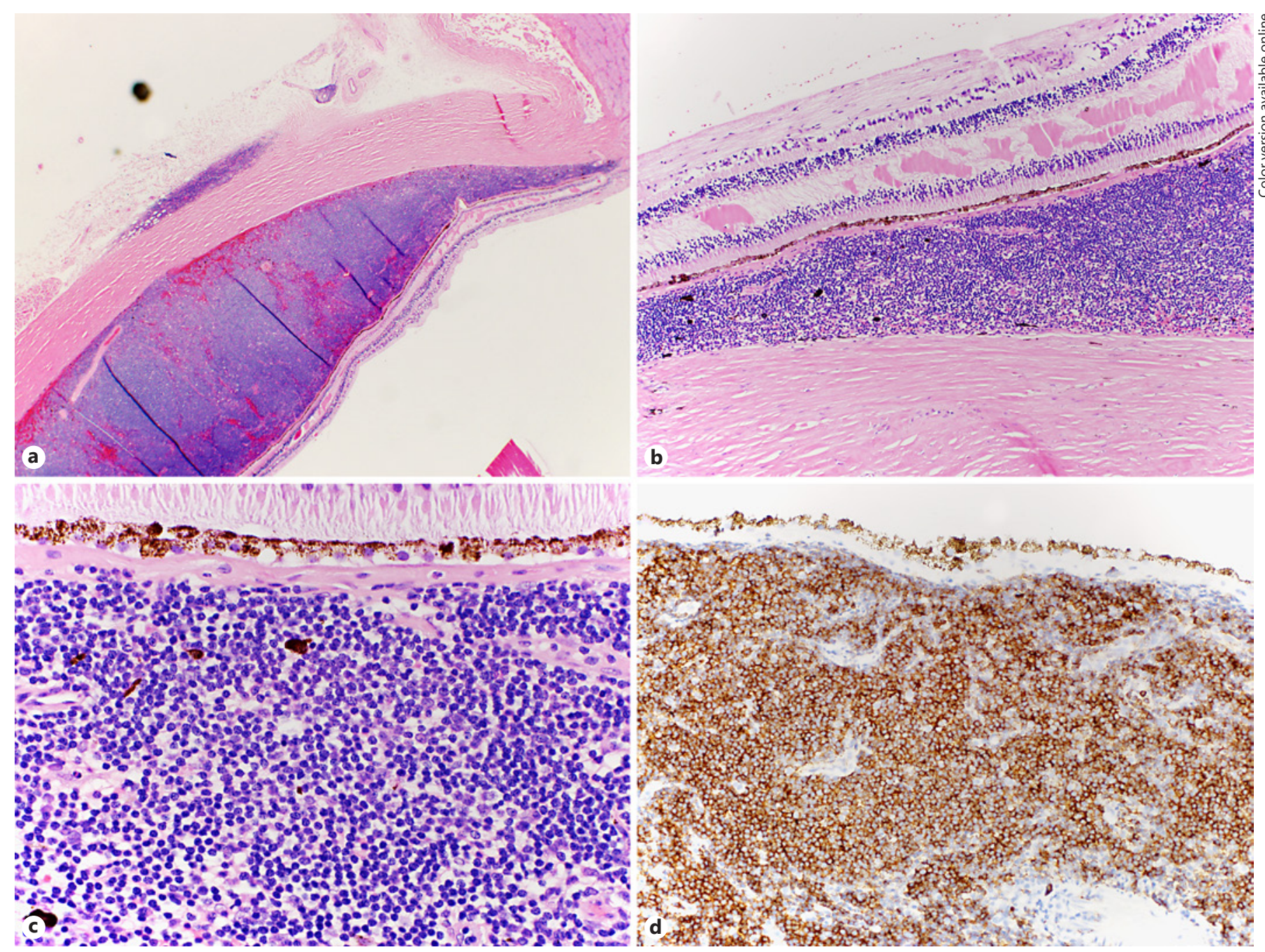

Fig. 3. Histopathology slides of enucleated left eye. a Diffuse choroidal and extraocular extension of lymphoma $(20 \times)$. b Choroidal infiltrates with retinal exudates $(100 \times)$. c Choroidal infiltrate with sheets of mature lymphocytes $(400 \times)$. d Positive CD20 staining of choroidal lymphoma $(400 \times)$.

pathologic analysis of the globe showed diffuse sheets of small lymphocytes with occasional cleaved-like cells and scattered plasma cells with many Mott cells and Dutcher bodies, replacing the choroid cells (Fig. 3). There was no iris or ciliary body involvement; however, the infiltrate extended to the extrascleral space tracking along the nerves. Small lymphocytes were diffusely positive for CD20, and were negative for CD5, CD23, lymphoid enhancer factor 1 (LEF1), BCL6, and BCL1. There was patchy staining with CD10 and CD43. Immunoglobulin light chain stains showed predominantly lambda expression over kappa. Overall, these findings were most consistent with extranodal marginal zone lymphoma. Evaluation for possible systemic involvement was recommended and the patient was referred to a medical oncologist. A CT scan of the brain was performed (an MRI could not be performed due to the patient's pacemaker) and showed possible sinus involvement. The patient was lost to further follow-up.

\section{Discussion}

This is an unusual presentation of a choroidal lymphoma in a patient suspected of possible corneal mass, which was in fact a descemetocele. This case underscores the value of B-scan ultrasonography when direct observation of the fundus is not possible, as well as the importance of histopathologic evaluation of all enucleated globes.

Extranodal marginal zone B-cell lymphoma, the most common form of uveal lymphoma, is derived from postgerminal cells and can be classified as mucosa-associated lymphoid tissue (MALT) lymphoma [5-8]. While CD10 positivity is somewhat unusual for marginal zone lym- 
phoma, rare cases have been described previously [9]. Immunohistochemical profile excluded other small B-cell lymphomas in the differential diagnosis including chronic lymphocytic leukemia/small lymphocytic lymphoma (CLL/SLL), follicular lymphoma, and mantle cell lymphoma. Consistent with previous reports, presentation was unilateral, and involved gradual visual changes with mild ocular surface discomfort [7]. Unlike many reported cases, the patient's vision loss was initially attributed to a corneal pathology, further masking suspicion of a posterior segment disease process. This case was also atypical in presenting with elevated intraocular pressure in the affected eye. In prior reports, ocular hypertension was present in $10 \%$ of choroidal lymphoma, and fewer for patients with primary than secondary lymphoma [3]. The ocular hypertension in this case was likely not due to lymphoma, but rather from angle and anterior segment scarring from prior HSV infection.

While enucleation was chosen due to blind and uncomfortable eye, external beam radiotherapy is the most common treatment for choroidal lymphoma, followed by observation, and systemic rituximab and other chemotherapeutic agents, with enucleation necessary in less than 1 in 20 cases [3].
Although the patient was lost to follow-up and systemic disease was unable to be ruled out, the unilaterality and low-grade subtype are suggestive of primary choroidal lymphoma. Previous studies have demonstrated low risk of patients developing systemic lymphoma if not identified at the time of diagnosis [3].

\section{Statement of Ethics}

The study complied with the guidelines for human studies. The subject gave informed consent for use of clinical images, and the case report was deemed exempt by the institute's committee on human research.

\section{Disclosure Statement}

The authors have no conflict of interest.

\section{Funding Sources}

Supported in part by That Man May See, Inc., an unrestricted grant from Research to Prevent Blindness, New York, NY, and grant EY002162 (Core Grant for Vision Research) from the National Eye Institute.

\section{References}

1 Aronow ME, Portell CA, Sweetenham JW, Singh AD: Uveal lymphoma: clinical features, diagnostic studies, treatment selection, and outcomes. Ophthalmology 2014;121:334341.

2 Coupland SE, Joussen A, Anastassiou G, Stein $\mathrm{H}$ : Diagnosis of a primary uveal extranodal marginal zone B-cell lymphoma by chorioretinal biopsy: case report. Graefes Arch Clin Exp Ophthalmol 2005;243:482-486.

3 Mashayekhi A, Shukla SY, Shields JA, Shields CL: Choroidal lymphoma: clinical features and association with systemic lymphoma. Ophthalmology 2014;121:342-351.
4 Sarafzadeh S, Corrêa ZM, Dhamija A, Augsburger JJ, Trichopoulos N: Intraocular lymphoma diagnosed by fine-needle aspiration biopsy. Acta Ophthalmol 2010;88:705-710.

5 Bardenstein DS: Orbital and adnexal lymphoma; in Singh AD, Damato BE, Pe'er J, et al. (eds): Clinical Ophthalmic Oncology. Philadelphia, PA: Saunders-Elsevier; 2007, pp 565570 .

6 Freeman C, Berg JW, Cutler SJ: Occurrence and prognosis of extranodal lymphomas. Cancer 1972;29:252-260.
7 Coupland SE, Damato B: Understanding intraocular lymphomas. Clin Experiment Ophthalmol 2008;36:564-578.

8 Coupland SE, Foss HD, Hidayat AA, Cockerham GC, Hummel M, Stein H: Extranodal marginal zone $\mathrm{B}$ cell lymphomas of the uvea: an analysis of 13 cases. J Pathol 2002;197:333340.

9 Wang E, West D, Kulbacki E: An unusual nodal marginal zone lymphoma with bright CD10 expression: a potential diagnostic pitfall. Am J Hematol 2010;85:546-548. 